\title{
The Beneficial Effect of Traditional Chinese Exercises on the Management of Obesity
}

\author{
Yuan Qin $\mathbb{D}^{1},{ }^{1}$ Weiyi Xia, ${ }^{1}$ Wei Huang, ${ }^{2}$ Jing Zhang, ${ }^{3}$ Yi Zhao, ${ }^{1}$ and Min Fang $\mathbb{D}^{1}$ \\ ${ }^{1}$ Shanghai University of Traditional Chinese Medicine, Shanghai, China \\ ${ }^{2}$ Shuguang Hospital Affiliated to Shanghai University of Traditional Chinese Medicine, Shanghai, China \\ ${ }^{3}$ Shanghai Green Valley Pharmaceutical Co., Ltd., Green Valley Research Institute, Shanghai, China \\ Correspondence should be addressed to Min Fang; fm-tn0510@shutcm.edu.cn
}

Received 27 June 2020; Revised 9 September 2020; Accepted 18 September 2020; Published 1 October 2020

Academic Editor: Xue-Qiang Wang

Copyright (C) 2020 Yuan Qin et al. This is an open access article distributed under the Creative Commons Attribution License, which permits unrestricted use, distribution, and reproduction in any medium, provided the original work is properly cited.

This paper systematically reviewed the clinical update of traditional Chinese exercises in the treatment of simple obesity in recent years and discussed their specific advantages in this aspect. This review focused on several typical traditional Chinese exercises, namely, Tai Chi, Ba Duan Jin, Yi Jin Jing, Wu Qin Xi, Shaolin Neigong, and Liu Zi Jue, which all showed clinical beneficial effect on the treatment of simple obesity with their own characteristics. To optimize the clinical therapeutic effect of these traditional Chinese exercises, we need to seek the most appropriate exercise or the combo exercise based on the characteristics of different obese population, to improve the efficiency of weight loss, reduce sports injury, and consolidate the therapeutic effect. In the future, we need to further evaluate the efficacy of sitting exercise, lying exercise, and static training in the treatment of simple obesity, subdivide the treatment population, and explore the working mechanism of these traditional Chinese exercises.

\section{Introduction}

Obesity is a clinical syndrome in which the body fat content is too high and/or the distribution is abnormal under the action of genetic and environmental factors, so that the actual body weight exceeds $20 \%$ of the ideal body weight and can be complicated with cardiovascular disease and a variety of endocrine and metabolic disorders [1].

Data from the Blue Book on Obesity Prevention and Control in China in 2019 show that the proportion of overweight and obesity in China is more than $40 \%$, and 10 to $20 \%$ of children are obese or overweight [2]. Obesity not only affects the external image but also causes psychological problems such as depression [3]. Obesity in adolescents can cause chronic brain hypoxia and affect learning. Obesity is also a risk factor for chronic diseases such as cardiovascular disease, diabetes, and some tumors [4].

According to the management guidelines of the American Obesity Society, the treatments for obesity include dietary intervention, lifestyle intervention, drug treatment, and surgery [5]. Due to some nonideal situation including poor patient compliance, many adverse reactions, and easy rebound, there is an urgent need for a healthier, simpler, and more sustainable treatment.

Exercise is a common way to lose weight, and scholars at home and abroad are exploring and establishing scientific and effective exercise way [6].

Traditional Chinese exercises are an ancient way of exercise and fitness, which have been reported to have unique advantages in the treatment of obesity. In this paper, we summarize the related research on the traditional Chinese exercises in the treatment of obesity in recent years to provide reference for clinical application and experimental research.

\section{Tai Chi}

Tai Chi is a form of sport that includes oriental culture, with the characteristics of both internal and external practice, firmness and softness, and slow and light spirit, which is the first batch of national intangible cultural heritage in our country [7]. Tai Chi is a medium- and low-intensity aerobic 
project [8]. A large number of studies have shown that Tai Chi has a significant effect in the treatment of the onset and progression of obesity.

Studies have found that Tai Chi exercise can gradually restore the normal activity of abnormally expressed AMPK genes in obese patients [9] and significantly reduce plasma neuropeptide Y [10], especially in patients with high triglycerides. Tai Chi can also improve the level of serum ghrelin in high-risk groups of metabolic syndrome [11], regulate the feeding center, reduce body weight, reduce waist circumference, improve blood lipid indexes, and eliminate the risk factors of metabolic syndrome.

The rapid proliferation of adipocytes caused by the proliferation and differentiation of preadipocytes is one of the possible mechanisms of obesity [12]. It has been found that the growth of hormone $\mathrm{GH}$ and catecholamine can inhibit the differentiation of preadipocytes in different degrees $[13,14]$. Long-term Tai Chi exercise can significantly increase the GH content of growth hormone [15], reduce the concentration of catecholamine, and increase the level of catechol statin [16]. In the study of lipid metabolism and related hormones in Tai Chi, it was found that after Tai Chi exercise, the indexes of total cholesterol (TC), triglyceride (TG), and low-density lipoprotein cholesterol (LDL C) decreased, while high-density lipoprotein cholesterol (HDL C) increased [17]. Tai Chi can significantly reduce the levels of serum insulin and leptin [18] and increase the level of adiponectin, a special active peptide secreted by adipocytes [19]. It can enhance insulin sensitivity and promote the oxidation of fatty acid in the body. It can inhibit inflammation to a certain extent [20]. Tai Chi exercise can reduce fasting blood glucose, 2-hour postprandial blood glucose, and glycosylated hemoglobin, stabilize human blood glucose level [21], and has a good balance effect on human energy metabolism.

A study found that after 6 months of Tai Chi exercise [22], the number of intestinal acidophilus, Lactobacillus, and Bifidobacterium increased significantly in obese elderly people and positively correlated with the training time. Through the effect on intestinal flora, the transformation and utilization of cholesterol was strengthened, and the level of blood lipids in the human body was ameliorated. In addition, Tai Chi exercise can also reduce lipid peroxidation, significantly reduce hs-CRP, IL- 6 , TNF- $\alpha$, and other inflammatory indicators, and ameliorate the microinflammatory status caused by obesity [23].

\section{Ba Duan Jin}

Ba Duan Jin has the advantages of softening the tendons and strengthening the bones, nourishing the qi and enhancing strength, promoting qi and invigorating blood circulation, and coordinating the functions of all the organs. After the reorganization by the State General Administration of Sports, the current version has a total of eight movements, each with a different focus, which can be practiced according to a certain viscera or disease syndrome [24].

Studies have shown that Ba Duan Jin exercise has a good effect on weight loss and good posture by losing weight and reducing waist and hip circumference [25]. Ba Duan Jin exercise could effectively reduce fasting blood glucose, serum insulin concentration, and insulin resistance index [26] and reduce the concentration of total cholesterol, triglycerides, and free fatty acid, and the level of leptin decreased with the decrease of blood lipid level and obesity index (body weight, BMI, and percentage of body fat), while adiponectin level was negatively correlated [27]. There is evidence showing that after practicing Ba Duan Jin, the number of Bifidobacterium, Bacteroides, and Lactobacillus increased significantly, while Enterobacter, Clostridium, and Enterococcus decreased significantly [28]. By regulating the structure of intestinal flora in the elderly, fasting-induced fat factor (fasting-induced adipocyte factor, FIAF) [29] can be affected and triglyceride deposition and storage can be reduced. The inflammatory markers (CRP, IL-6, and TNF- $\alpha$ ) have been found decreased significantly in Ba Duan Jin participants [30]. However, its inhibitory effect on obesity still needs to be verified by further experiments.

\section{Yi Jin Jing}

Yi Jin Jing is a fitness exercise characterized by improving the function of muscles and bones, stimulating the circulation of the blood, and causing the muscles and joints to relax. After the reorganization by the State General Administration of Sports, the current version has a total of 12 postures, with the combination of movement and stillness. The movement requires stretching tendons and bones, fully flexion and extension, abduction, adduction, torsion of the body, etc., in order to pull the muscles and fascia of various parts of the human body, as well as the tendons, ligaments, joint capsules, and other connective tissues, which can play a positive role in body shape and physiological function. Persistent exercise can strengthen the muscles and bones and benefit the internal viscera [31].

Practicing Yi Jin Jing can also regulate glucose and lipid metabolism. The study found that 6 months of Yi Jin Jing exercise can significantly reduce the levels of serum TC, TG, and LDL C and increase the level of HDL C [32]. Long-term persistence can reduce the levels of fasting blood glucose and insulin and reduce insulin resistance accompanied by the decrease of inflammatory reactions [33]. The percentage of CD4+ T cells, CD8+ T cells, and NK cells and the levels of IL2 , IL- 6 , and IFN- $\gamma$ in the immune indexes of the elderly were significantly increased after 24 weeks of practicing Yi Jin Jing [34], so as to improve the immunity of the elderly.

\section{Wu Qin Xi (Five Fowl Opera)}

$\mathrm{Wu}$ Qin Xi is a set of traditional aerobics exercise created by Hua Tuo, a famous physician of the Eastern Han Dynasty, who combined the posture and movements of the tiger, deer, bear, bird, and ape, with the theory of the five elements of traditional Chinese medicine. It is the earliest guiding technique recorded so far and is the world-class intangible cultural heritage [35].

$\mathrm{Wu}$ Qin Xi exercise could reduce the serum levels of TC, TG, and LDL C and increase the level of HDL C in elderly 
women and significantly improve the level of blood lipids after 6 months of practice [36]. After 24 weeks of practice of $\mathrm{Wu}$ Qin Xi, blood sugar and glycosylated hemoglobin levels decreased by $7.14 \%$ and $13.23 \%$, respectively, which effectively controlled blood sugar levels [37]. At the same time, $\mathrm{Wu}$ Qin Xi practice can also reduce the levels of leptin and insulin and increase the level of adiponectin [38, 39]. After practicing Wu Qin Xi for 2 months [40], the amount of Bifidobacterium, Lactobacillus, Bacteroides, Clostridium, SCFAs, GLP-1, and GLP-2 in the intestine of the practitioners increased, and this effect increased with time. $\mathrm{Wu}$ Qin Xi reconditioned human microecology, improved the structure of gut microbiota, reduced intestinal permeability, and regulated human metabolism. Wu Qin Xi practice improved the immune function of the middle aged and elderly [41, 42]. It was found that the activity of NK cells and the ratio of $C D+4 / C D+8$ increased significantly after exercise, which played a positive role in regulating immune balance.

\section{Shaolin Neigong}

Shaolin Neigong is an important part of internal Qigong massage therapy, that is, Shanghai intangible cultural heritage. This exercise pays attention to strength and endurance with the lower limb standing crotch posture as the basic exercise combining with the upper limb movement. It is characterized by skeletal muscle static contraction exercise [43], emphasizing the "static resistance" of the lower limbs and the "internal strength" of the upper limbs [44].

The effective rate of Shaolin Neigong in the treatment of simple obesity among college students is $85.7 \%$, which can significantly reduce the weight, BMI, and waistline and hip circumference of practitioners [45]. Shaolin Neigong can reduce the insulin resistance index and improve the sensitivity of the body to insulin [46]. The exercise frequency test of Shaolin Neigong showed that when the exercise frequency was 1-4 times a day, the subjects' levels of FPG, 2hPBG, and HbAlc were improved [47].

It was found that the inverted suspension of rats could simulate the static characteristics of Shaolin Neigong [48]. It was found that static training could increase the transcription level of the hypothalamic POMC gene and the content of $\beta$-endorphin in the hypothalamus [49], thus reducing the appetite for food. A study reported that static training combined with massage therapy could increase the protein content of skeletal muscle, reduce the excretion of creatinine (CRE) and 3-methylhistidine (3-MH), and regulate protein metabolism [50]. Qigong training can partially eliminate the RNA interference of the PGC- $1 \alpha$ signal pathway [51], suggesting that this kind of static training can facilitate the activation of the PGC- $1 \alpha$ signal pathway, induce the expression of PGC- $1 \alpha$, and consequently regulate central appetite.

\section{Liu Zi Jue}

Liu Zi Jue refers to a breathing practice of exhale and inhale in six different ways, namely, si, $x u, x i, h e, h u$, and chui, in order to mobilize the function of various organs to activate qi and blood circulation. It is an ancient health-preserving method, which can make the breath natural, relax the body, and calm the mind.

Comparing the effects of different traditional exercises on fasting blood glucose, it showed that after 3 months of exercise, Wu Qin Xi, Ba Duan Jin, and Liu Zi Jue all could effectively reduce fasting blood glucose (FPG), and among them Liu Zi Jue showed the strongest effect [52]. After practicing Liu $\mathrm{Zi}$ Jue [53], both body weight and BMI showed a trend of decrease, and the grip strength, bouncing strength, fast walking, flexibility, and other exercise abilities of elderly were all improved in varying degrees.

\section{Others}

Comparing the effects of Tai Chi, Baduanjin, Yi Jin Jing, and Wuqinxi on the immune function of the elderly showed that they all can significantly increase the immune indexes, including $\mathrm{CD} 3+, \mathrm{CD} 4+, \mathrm{CD} 8+$, and NK cells. Among them, Tai Chi was the best in improving CD4+ and NK cells, and $\mathrm{Ba}$ Duan Jin was the best in improving CD3+ and CD8+ cells [54]. Both "Mawangdui Dao Yin" and fitness Qigong "Da Wu" can reduce TG, TC, and LDL C and increase HDL C to some extent $[55,56]$. Some scholars combined Mawangdui Dao Yin, Tai Chi, Shi Er Duan Jin, Dao Yin health-preserving gong, and $\mathrm{Da} \mathrm{Wu}$ to sort out a new set of Qigong exercise [57] and found that this exercise also can regulate lipid metabolism. In the study of the Pigu weight loss experiment, it showed a significant effect on weight loss [58].

Other traditional Chinese exercises that have not been verified by experiments, such as "weight loss and antiaging Qi gong” [59], Huichun Gong [60], Tiaoxi Zhuji Gong [61], Xiaoyao walking, Guanyin Gong, stepping abdomen beating Gong, abdominal retraction Gong, palm closing Gong [62], simple weight loss Gong, relaxation weight loss Gong, Xiao Zhou Tian loss Gong, Yannian Jiuzhuan Gong, Jade toad Xi Zhen Gong, Guanyin lotus seat, Jade toad wave turning Gong, standing pile, Longmen bodybuilding, and longevity Gong [63], are clearly stated that they have a certain degree of effect on weight loss. It is worth further exploring.

\section{Summary}

At present, there are many studies on the traditional Chinese exercises in the treatment of obesity, which reveal good results from different angles. Among these exercises, it seems that Tai Chi is the most widely studied one. In terms of the research direction, they are mostly focused on the effect of Qigong exercises on the level of glucose and lipid metabolism, but less on central feeding regulation, insulin, leptin, and adiponectin. In recent years, gut microbiota and immune functional regulation have attracted more attention. Compared with modern medicine, traditional Chinese medicine pays attention to personalized therapy based on the difference of patients and their different syndromes. In terms of obesity, it has the characteristics of wide age span, different degrees of obesity, and always has one or more complications. The traditional Chinese exercises mentioned 
in this paper not only have the commonness of weight loss and fat reduction, but also have their own characteristics, which can be selectively used for different types of obese patients.

Slow movement with breathing is what the above traditional methods have in common. When practicing Tai Chi, $\mathrm{Ba}$ Duan Jin, and Wu Qin Xi, the big movements of the upper and lower limbs cooperate with breathing. Tai Chi is beneficial to the overall balance, and Ba Duan Jin can increase lower limb strength and muscle content [64] and reduce fat content [65]. Wu Qin Xi can improve flexibility. The improvement of the heart rate of Tai Chi is better than that of Wu Qin Xi [66], while the exercise intensity is lower than that of Ba Duan Jin and Wu Qin Xi. For example, Tai Chi is suitable for patients with mild to moderate obesity, especially for the middle aged and elderly because Tai Chi belongs to low-intensity exercise and has advantages in controlling blood pressure, increasing bone mineral density, and reducing blood sugar, but it is not suitable for patients with severe obesity or knee osteoarthritis. Ba Duan Jin is more suitable for obese patients with endocrine and digestive system diseases such as cervical spondylosis and perimenopausal syndrome. Wu Qin Xi has certain advantages in improving the hyperlipidemia of adult obese patients.

The standing pile and movement of Yi Jin Jing Sutra pay attention to the stretching of muscles, standing pile after completing the action, and stretching when inhaling and relaxing when exhaling, which belong to intermittent-, medium-, and low-intensity training that can increase the muscle strength of the lower limbs of the elderly [67]. The standing pile and action of Shaolin Neigong emphasize the static contraction of muscles. Both the standing pile and the big movement should include contracting the muscles hard, breathing naturally, completing the action after standing pile, relaxing the whole body, and then continuing to the next standing pile or action after a short rest, which belongs to intermittent-, medium-, and high-intensity training. Shaolin Neigong reduces appetite and increases skeletal muscle content. Yi Jin Jing can slow down the physical decline and improve the symptoms of constipation. Shaolin Neigong has the characteristics of intermittent-, medium-, and high-intensity exercise, which is especially suitable for obese patients who are not easy to exercise because of excessive weight and also fit for the obese patients with poor cardiopulmonary function.

Liu $\mathrm{Zi} \mathrm{Jue} \mathrm{can} \mathrm{improve} \mathrm{human} \mathrm{body} \mathrm{function} \mathrm{through}$ breathing exercises, slow down heart rate, increase vital capacity, stimulate vegetative nervous system, promote venous reflux, change hemodynamics, reduce hemorheological indexes, and improve the motility of visceral organs [68]. Liu $\mathrm{Zi}$ Jue is most suitable for obese patients with chronic obstructive pulmonary disease.

In addition, the sitting and lying exercises in the traditional method have also their own features, which are especially suitable for patients who cannot practice standing up; however, it still needs further study.

The traditional Chinese exercises have a long history, which contain the Chinese traditional fitness culture and also a concrete practice of the concept of "preventive treatment of disease" in traditional Chinese medicine. These exercises not only reduce fat and weight and adjust posture but also cultivate physical and mental health, which is beneficial. It is a simple and effective way to lose weight with low cost and is environment friendly.

\section{Data Availability}

This article is a review article and does not contain relevant data.

\section{Conflicts of Interest}

The authors declare that there are no conflicts of interest regarding the publication of this paper.

\section{Authors' Contributions}

Yuan Qin, Weiyi Xia and Wei Huang contributed equally to this work.

\section{Acknowledgments}

The authors would like to thank Professor Xue Qiang Wang, Shanghai University of Sport, for assistance with drafting the paper. This study was financially supported by Project 2018 of the Science and Technology Commission of Shanghai (no. $18 \mathrm{dz2308400)}$ and by the Shanghai Municipal Health Commission (no. ZY (2018-2020)-CCCX-2001-05). The authors are grateful to all participants who took part in the study.

\section{References}

[1] X. F. Yu, Guidelines for Diagnosis and Treatment of Endocrine and Metabolic Diseases, Science Press, Beijing, China, 3rd edition, 2013.

[2] Y. F. Wang, M. Sun, and Y. Yang, Blue Book on Obesity Prevention and Control in China Version 1, Peking University Medical Press, Beijing, China, 2019.

[3] Y. Wu, J. D. Li, S. P. Zhu et al., "The harm and treatment status of adolescent-obesity," Chinese Electronic Journal of Obesity and Metabolic Diseases, vol. 3, no. 2, pp. 70-73, 2017.

[4] J. Hong, "The current situation, harm and coping strategies of obesity in China," in Proceedings of the 12th National Conference on Endocrinology of Chinese Medical Association, Xi'an, China, 2013.

[5] J. L. Cao and X. Z. Lu, "2013 interpretation of American heart association/American college of cardiology/American obesity association guidelines on adult overweight and obesity," Chinese Journal of Hypertension, vol. 22, no. 9, pp. 813-816, 2014.

[6] B. Bu, "Research progress of exercise prescription," Chinese Journal of Evidence-Based Medicine, vol. 10, no. 12, pp. 1359-1366, 2010.

[7] Y. J. Wu, "Research on the inheritance and development of traditional Taijiquan," Shaolin and Taiji (Zhongzhou Sports), vol. 2, pp. 5-7, 2012.

[8] Y. Q. Zhu, Z. Y. Shen, P. Yu et al., "A dynamic study on the level of aerobic exercise in the process of practicing Taijiquan 
among people of different ages," Shanghai Journal of Traditional Chinese Medicine, vol. 46, no. 2, pp. 7-9, 2012.

[9] Y. Qian and J. B. Ke, "Effect of Taijiquan exercise on whole genome expression of obese patients," Journal of the Institute of Military Physical Education, vol. 29, no. 3, pp. 120-122, 2010.

[10] J. H. Wang and Y. Cao, "Effect of Taijiquan exercise on neuropeptide $\mathrm{Y}$ in elderly obese patients with type 2 diabetes mellitus," Sports and Science, vol. 4, pp. 67-68, 2003.

[11] Y. B. Han, "Effect of Taijiquan training on growth hormone releasing peptide and adiponectin in high risk population of metabolic syndrome," Abstract Compilation of Papers of the Ninth National Sports Science Congress, China Sport Science Society, Beijing, China, 2011.

[12] I. M. Faust, P. R. Johnson, J. S. Stern et al., "Diet-induced adipocyte number increase in adult rats: a new model of obesity," American Journal of Physiology, vol. 235, no. 3, pp. 279-286, 1978.

[13] H. J. Zhu, Effects of IGF-1, GH and Ghrelin on Proliferation and Differentiation of Preadipocytes, China Union Medical University, Beijing, China, 2003.

[14] X. H. Zhu, Q. L. He, and Z. H. Lin, "Effect of catecholamine on proliferation and differentiation of human preadipocytes," Chinese Journal of Plastic Surgery, vol. 19, no. 4, pp. 282-284, 2003.

[15] M. Y. Wang, "Effects of long-term Taijiquan exercise on serum testosterone/cortisol, immunoglobulin and growth hormone in elderly women," Journal of Capital Institute of Physical Education, vol. 21, no. 3, pp. 343-345, 2009.

[16] M. Z. Yuan, H. H. Xue, and Y. M. Huang, "The influence of 20-week Taijiquan exercise on the physical function of college students," Journal of Military Sports, vol. 36, no. 3, pp. 85-88, 2017.

[17] Z. Y. Jin, "Effect of Taijiquan exercise on hypertension and blood lipids in the middle-aged and elderly," Modern Preventive Medicine, vol. 39, no. 17, pp. 4468-4469, 2012.

[18] H. L. Zhang and H. J. Zhang, "Effects of long-term practice of Taijiquan on Lipid Metabolism and related hormones in obese College students," Journal of Shenyang Institute of Physical Education, vol. 30, no. 6, pp. 95-98, 2011.

[19] Q. Jia and L. L. Zhu, "Study on Taijiquan combined with psychological intervention to improve risk factors and adiponectin in patients with metabolic syndrome," Journal of Cardiovascular Rehabilitation Medicine, vol. 19, no. 4, pp. 337-339, 2010.

[20] Z. Dastani, M. F. Hivert, N. Timpson et al., "Novel loci for adiponectin levels and their influence on type 2 diabetes and metabolic traits: a multi-ethnic meta-analysis of 45891 individuals," PLoS Genetics, vol. 8, no. 3, 2012.

[21] J. W. Zhang, "Comparative study on the effect of Taijiquan exercise on health intervention in patients with type 2 diabetes mellitus of different genders," Abstract Compilation of Papers of the 11th National Sports Science Congress, China Sports Science Society, Beijing, China, 2019.

[22] T. Zhou, Z. Z. Qiu, and W. Liu, "Study on the effect of Taijiquan exercise on intestinal probiotics and Blood Lipid Metabolism in obese elderly," Journal of Shandong Institute of Physical Education, vol. 28, no. 1, pp. 62-66, 2012.

[23] X. B. Li, "Effects of Taijiquan exercise on oxidative stress and inflammation in elderly patients with type 2 diabetes mellitus," Chinese Journal of Gerontology, vol. 33, no. 21, pp. 5465-5466, 2013.

[24] H. F. Qiu, Baduanjin, Jilin Science and Technology Press, Changchun, China, 2009.
[25] X. M. Pang and H. S. Pan, "Investigation and analysis of Baduanjin on exercise weight loss of obese female college students," Health Care Guide, vol. 21, pp. 293-294, 2016.

[26] M. H. Sun, "Effect of fitness Qigong Baduanjin exercise on indexes of patients with type 2 diabetes mellitus," Chinese Sports Coaches, vol. 26, no. 1, pp. 49-55, 2018.

[27] Y. Zhou, C. Fan, Y. B. Chen et al., "Effects of Baduanjin exercise combined with calcium pyruvate supplementation on serum leptin and adiponectin in obese female college students," Liaoning Sports Science and Technology, vol. 35, no. 6, pp. 26-28, 2013.

[28] H. M. Sun, "Effect of fitness Qigong Baduanjin exercise on intestinal flora in the elderly," Chinese Journal of Sports Medicine, vol. 31, no. 11, pp. 973-977, 2012.

[29] F. Bäckhed, J. K. Manchester, C. F. Semenkovich, and J. I. Gordon, "Mechanisms underlying the resistance to dietinduced obesity in germ-free mice," Proceedings of the National Academy of Sciences, vol. 104, no. 3, pp. 979-984, 2007.

[30] Q. Li, Q. L. Yao, X. Q. He et al., "Observation on the efficacy of Baduanjin exercise prescription in improving the state of microinflammation in patients with maintenance peritoneal dialysis," Chinese Journal of Nephropathy of Integrated Traditional Chinese and Western Medicine, vol. 21, no. 2, pp. 160-161, 2020.

[31] G. Du, Yi Jin Jing, Tianjin People's Art Publishing House, Tianjin, China, 2016.

[32] M. Yuan, "Effect of fitness Qigong Yijin meridian on blood lipid in patients with hyperlipidemia and its mechanism," Nanjing University of Traditional Chinese Medicine, Doctoral dissertation, Nanjing, China, 2014.

[33] D. S. Meng, "Experimental study on the therapeutic effect of traditional fitness function method on type 2 diabetes mellitus," Guangxi Normal University, Doctoral dissertation, Guilin, China, 2017.

[34] Y. C. Feng, G. L. Zhong, W. M. Qiu et al., "Effect of fitness Qigong Yijin meridian on immune function of the elderly," Journal of Jilin Institute of Physical Education, vol. 29, no. 5, pp. 68-70, 2013.

[35] The State General Administration of Sports, Fitness Qigong Five Fowl Play, People's Sports Publishing House, Beijing, China, 2003.

[36] P. Sha, "Effect of fitness Qigong Wuqin Opera on blood lipid indexes and balance ability of middle-aged and elderly women," Shanxi Traditional Chinese Medicine, vol. 31, no. 10, pp. 1332-1335, 2010.

[37] X. F. Hu, "Effect of poultry opera exercises on glucose metabolism in elderly female patients with T2DM on Friday," Abstract Compilation of Papers of the 10th National Sports Science Congress of Chinese Sports Science Society, China Sports Science Society, Beijing, China, 2015.

[38] R. Q. Fang, "Effect of characteristic teaching mode of traditional Chinese medicine in Wuqinxi on serum leptin and insulin resistance of obese senior high school students," Journal of Jinzhou Medical University, vol. 35, no. 5, pp. 26-30, 2018.

[39] H. M. Sun, "Effect of Wuqian Opera on metabolic syndrome in middle-aged men and its biological mechanism," China Sports Science and Technology, vol. 51, no. 4, pp. 86-92, 2015.

[40] H. M. Sun and Y. P. Zhong, "Intervention study of Wuqinxi on intestinal flora and its metabolites in middle-aged male patients with metabolic syndrome," Journal of Chengdu Institute of Physical Education, vol. 45, no. 3, pp. 81-87, 2019.

[41] D. H. Yu and J. H. Wang, "Changes of T lymphocytes in peripheral blood of middle-aged and elderly before and after 
6-month Qigong five-fowl play exercise," Chinese Journal of Sports Medicine, vol. 2, pp. 206-270, 2007.

[42] D. H. Yu and J. H. Wang, "Changes of NK cell activity before and after 3 months of Wuqian play exercise in the middleaged and elderly," Chinese Journal of Sports Medicine, vol. 5, pp. 602-603, 2005.

[43] A. L. Wang and D. S. Liu, "Methods of strength training: static strength training, centripetal strength training and eccentric strength training," Chinese School Physical Education, vol. 1, no. 8, pp. 67-71, 2014.

[44] Q. W. Li and Q. M. Li, "Massage veteran doctor Li Xijiu's Shaolin internal exercise method," Shanghai Journal of Traditional Chinese Medicine, vol. 10, pp. 31-33, 1983.

[45] H.-x. Lü, Z.-q. Wu, S.-f. Zhang et al., "Effect of Tuina exercise on simple obesity in college students," Journal of Acupuncture and Tuina Science, vol. 16, no. 6, pp. 394-401, 2018.

[46] T. Zhang, F. C. Lin, Z. H. Fang et al., "Effect of traditional Chinese medicine on insulin resistance in patients with prediabetes," Chinese Journal of Gerontology, vol. 38, no. 22, pp. 5419-5421, 2018.

[47] S. Yu, "Clinical observation of 24-week fitness Shaolin internal exercise intervention in patients with prediabetes," Nanjing University of Traditional Chinese Medicine, Doctoral dissertation, Nanjing, China, 2016.

[48] P. Wan, J. Xu, J. T. Yan et al., "A simple static training method for rats," Chinese Journal of Sports Medicine, vol. 14, no. 2, p. $61,1998$.

[49] H. Zhang, J. T. Yan, J. Xu et al., "Effect of static training on $\beta$-endorphin and POMC gene expression in rats," Journal of Shanghai University of Traditional Chinese Medicine, vol. 1, pp. 44-46, 2003.

[50] H. Zhang, J. T. Yan, J. Xu et al., "Effect of static training combined with massage therapy on protein metabolism in aged rats," Journal of Shanghai University of Traditional Chinese Medicine, vol. 1, pp. 35-37, 2004.

[51] Z. R. Li, L. Fang, W. B. Wu et al., "To study the effect of static exercise on upper limb motor ability of aged rats based on RNA interference of PGC- $1 \alpha$ signal pathway," Shi Zhenguo Medical Medicine, vol. 30, no. 1, pp. 247-249, 2019.

[52] Y. Z. Wang, "Effects of different fitness Qigong on fasting blood glucose and fructosamine in patients with type 2 diabetes mellitus," Contemporary Sports Science and Technology, vol. 7, no. 30, pp. 247-248, 2017.

[53] S. J. Sun, X. T. Zhen, and R. S. Tu, "The influence of the sixcharacter formula of fitness Qigong on the body shape, function, quality and exercise ability of the middle-aged and elderly," International Journal of Traditional Chinese Medicine, vol. 11, pp. 985-987, 2011.

[54] L. P. Liang, "Effects of a variety of traditional health care sports on psychological emotion and immune function of the middle-aged and elderly," Chinese Journal of Gerontology, vol. 38, no. 2, pp. 418-420, 2018.

[55] B. Wang, Z. K. Wu, S. T. Lu et al., "Effect of body-building Qigong Mawangdui guided exercise on blood lipid metabolism and free radical metabolism in middle-aged and elderly women," Chinese Journal of Geriatrics, vol. 34, no. 13, pp. 3720-3722, 2014.

[56] X. Y. Zhang, Experimental Study on the Effect of Fitness Qigong Dance on Improving the Sub-Health Status of Middle-Aged and Elderly, Shenyang Institute of Physical Education, Shenyang, China, 2014.

[57] C. K. Li, H. B. Dai, M. Xu et al., "Effect of new Qigong method on blood lipids in middle-aged and elderly," Journal of
Chengdu Institute of Physical Education, vol. 39, no. 9, pp. 62-66, 2013.

[58] Q. X. Wang, S. Guo, and Y. B. Sun, "Study on the effect of four valley-clearing methods on weight loss," Education and Teaching Forum, vol. 21, pp. 264-265, 2013.

[59] X. N. Li, "Qigong method of obesity," Journal of Traditional Chinese Medicine, vol. 8, p. 51, 1992.

[60] T. D. Wang and M. Shen, "Huichungong achieved good results in losing weight," Chinese Qigong, vol. 6, pp. 16-17, 1990.

[61] S. F. Song, "Clinical observation on 180 cases of weight loss by bodybuilding Qigong," Journal of Qigong, vol. 3, pp. 113-114, 1998.

[62] J. Yi, "Exercise of Qigong weight loss method," Shanghai Journal of Traditional Chinese Medicine, vol. 8, 2020.

[63] G. F. Zhang and J. B. Huang, Treatment of Obesity with Traditional Chinese Medicine, Southwest Normal University Press, Chongqing, China, 2015.

[64] Q. X. Xie, "Comparative study of fitness function between Taijiquan and Qigong Baduanjin," Journal of Changchun Normal University, vol. 34, no. 4, pp. 78-81, 2015.

[65] Y. Jiang, Z. Q. Lai, K. L. Fan et al., "Effects of 12-week health Qigong Baduanjin on lower limb body compositions in knee Osteoarthritis patients," Journal of Liaoning University of Traditional Chinese Medicine, vol. 22, no. 8, pp. 90-93, 2020.

[66] F. W. Dong and R. Q. Chang, "Comparative research on the effect of Taijiquan and Wuqinxi to college students' body fitness," Bulletin of Sport Science and Technology, vol. 25, no. 5, pp. 91-93, 2017.

[67] L. Gong, J. T. Yan, Y. C. Liu et al., "Effects of Sinewsstrengthening training on isokinetic muscle strength in Old patients with Sarcopenia," Journal of Shanghai University of Traditional Chinese Medicine, vol. 25, no. 3, pp. 55-58, 2011.

[68] X. L. Sun, "Nursing research on metabolism of glucose and lipid and hemorheology of the diabetic Nephropathy patients with Liuzijue-Qigong," Journal of Liaoning University of Traditional Chinese Medicine, vol. 20, no. 7, pp. 148-151, 2018. 